\title{
Influenza pandémica A (H1N1) 2009: epidemiología, características clínicas y diferencias con influenza estacional en Chile
}

\author{
Ricardo Rabagliati B., Leonardo Siri Z., Carlos M Pérez C., Jaime Labarca L. y Marcela Ferrés G.
}

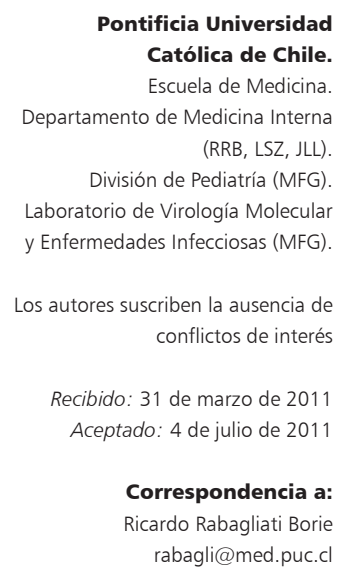

rabagli@med.puc.cl

\begin{abstract}
Pandemic influenza A (H1N1) 2009: epidemiology, clinical features and differences with seasonal influenza in Chile

Pandemic influenza A (H1N1) 2009 raised questions regarding differences with seasonal influenza. Objectives: To describe the clinical features of pandemic influenza and compare them to seasonal influenza. Patients y Methods: A descriptive study that compared hospitalized adults was done between patients with confirmed pandemic influenza in the Hospital Clínico Universidad Católica in Santiago, Chile, from May to July 2009 and 95 confirmed historic cases of seasonal influenza. Results: 54 patients with pandemic influenza were included, $51.9 \%$ were male, age of $52.8 \pm 19.5$ years old; $79.6 \%$ had chronic diseases; $16.7 \%$ were immunocompromised patients and $7.4 \%$ of pregnant women. $25.9 \%$ of the patients acquired the infection during the hospitalization. $31.5 \%$ were admitted to intermediate/intensive care units. Pneumonia was diagnosed in $37 \%$, and the mortality rate was 3.7\%. The comparison between pandemic and seasonal influenza showed less proportion of patient $\geq 65$ years of age $(31.5 \%$ vs. $68 \%$; $<0.0001)$; double number of nosocomial acquisition and more cases of pneumonia and death. Conclusions: The pandemic influenza infection affected younger people and was related with more nosocomial cases, pneumonia and mortality rates than seasonal influenza.

Key words: Pandemic influenza, A (H1N1), seasonal influenza.

Palabras clave: Influenza pandémica, A (H1N1), influenza estacional.
\end{abstract}

\section{Introducción}

A fines de abril del año 2009, se anunció un brote de influenza en México causada por un nuevo virus de influenza A (H1N1) de origen porcino, que no había infectado seres humanos previamente ${ }^{1}$. Se generó alarma internacional ante el hecho de que el virus se estaba propagando fácilmente de un país a otro. En Chile, el primer caso se detectó el 17 de mayo de $2009^{2}$ y al mes siguiente, al cumplirse los criterios de definición, fue declarada una pandemia por parte de la Organización Mundial de la Salud.

Los primeros informes desde México y E.U.A. describían una alta tasa de hospitalización, en especial en unidades de cuidados intensivos debido a neumonía, con mortalidad de hasta $9 \%{ }^{1}$. Estudios posteriores, que incluyeron descripciones de los casos con y sin confirmación de laboratorio ${ }^{3-5}$, corroboraron una alta tasa de complicaciones, aunque con menor mortalidad, de $0,6 \%$ a $0,001 \%{ }^{6,7}$. En el curso de la pandemia se hizo evidente el desconocimiento del real comportamiento del virus A (H1N1) en relación a los diferentes grupos de pacientes, el grado de transmisibilidad, el rendimiento de las pruebas de diagnóstico y las diferencias con la influenza estacional. En los primeros informes, era sorprendente ver que la influenza pandémica $A$ (H1N1) era menos frecuente en las personas mayores ${ }^{1,6} \mathrm{y}$ presentaba mayor gravedad en individuos jóvenes sanos ${ }^{6}$, características que no correspondían a los cánones clásicos de influenza estacional que tiene un impacto más grave en pacientes ancianos, con co-morbilidades e inmunocomprometidos ${ }^{8}$.

El presente estudio pretende contribuir con algunas respuestas a estas preguntas a través de la descripción clínica y epidemiológica del comportamiento de la infección, incluyendo casos de infección documentada por virus influenza A (H1N1) en población adulta que requirió hospitalización y realizar una comparación con casos históricos confirmados de influenza estacional en adultos hospitalizados en nuestra institución durante dos temporadas de influenza de 5 y 10 años previos.

\section{Pacientes y Métodos}

Estudio descriptivo realizado en el Hospital Clínico Universidad Católica de Chile en Santiago.

Procedimientos: De acuerdo a las normativas locales basadas en guías del Ministerio de Salud ${ }^{9}$, se definió como caso sospechoso al paciente con síntomas de influenza 
como fiebre superior a $38^{\circ} \mathrm{C}$ y/o síntomas tales como tos, mialgias, cefalea u odinofagia. La confirmación del virus de la influenza pandémica A (H1N1) se realizó mediante reacción de polimerasa en cadena (RPC) en todos los pacientes sospechosos que ingresaron para hospitalización desde el servicio de urgencia, o para aquellos pacientes hospitalizados por otra causa, que desarrollaron fiebre de cualquier grado y/o tuvieron uno o más de los síntomas descritos durante su estadía. Cada uno de los casos sospechosos fue manejado con precaución de contacto más gotitas mientras se obtenía la confirmación diagnóstica e iniciaron inhibidores de neuraminidasa, oseltamivir (75 mg dos veces al día) o zanamivir (10 mg dos veces al día). Caso confirmado: Se definió como un paciente sospechoso con RPC positiva; en estos casos, la terapia antiviral se continuó durante cinco días y se informó a los investigadores los cuales evaluaron el caso y recopilaron la información desde la ficha clínica (Figura 1).

Criterios de inclusión y exclusión: Se incluyeron solamente pacientes hospitalizados con edad $\geq 15$ años con diagnóstico confirmado de influenza pandémica A(H1N1), durante un período de nueve semanas, desde el 18 de mayo hasta el 19 de julio del año 2009. Los pacientes sintomáticos con una prueba de RPC negativa fueron excluidos. Los pacientes diagnosticados con influenza pandémica A (H1N1) que se derivaron desde otros hospitales para apoyo de ventilatorio debido a insuficiencia respiratoria grave tampoco fueron incluidos.

Comparación con influenza estacional: Los casos de influenza pandémica A (H1N1) se compararon con un total de 95 casos de pacientes adultos hospitalizados con diagnóstico de influenza estacional confirmada por laboratorio, recolectados durante el período de influenza estacional de 10 y 5 años anteriores, cuya caracterización clínica y epidemiológica había sido publicado previamente $^{10,11}$. Se incluyeron 50 adultos de 1999 y 40 del 2004, ingresados en el mismo centro y manejados por el mismo equipo de Infectología (RR, CP, JL). El virus influenza que circuló en ambas temporadas de influenza fue influenza A $(\mathrm{H} 3 \mathrm{~N} 2)^{12,13}$.

Muestras clínicas y técnicas de laboratorio: La muestra clínica fue obtenida por hisopado nasofaríngeo y para la confirmación se utilizó una técnica comercial de RPC en tiempo real proporcionada por Roche Diagnostics. La técnica incluye dos fases de amplificación para el diagnóstico. La primera parte detecta un fragmento de $250 \mathrm{pb}$ del gen de la influenza A M2, que confirma la presencia del virus de influenza A (Light Mix Kit Influenza A Virus M2, Cat $n^{\circ}$ 40-0234-16 TIB Mol Biol). La segunda parte determina si corresponde a virus H1 "porcino", con cebadores específicos de la cepa H1 (SOIV), un fragmento de $123 \mathrm{pb}$ de la secuencia del gen influenza A (Light Mix Kit Inf A swine H1 ${ }^{\circledR}$, cat- $N^{\circ}$ 40-0580-32, TIB, Mol Biol). El
LightCycler instrument versión 2.0 elaborado por Roche Diagnostics se utilizó para ambos pasos.

A solicitud del médico tratante, previo a la realización de la RPC confirmatoria de influenza A (H1N1), en algunos casos se obtuvo muestras clínicas de hisopado nasofaríngeo para inmunofluorescencia directa para influenza A (Diagnostic Hybrids, Athens Ohio USA) o inmunocromatografía (quickvue Influenza A+B test, Quidel ${ }$, San Diego CA).

Análisis estadístico: Se creó una base de datos para el análisis descriptivo. Los resultados obtenidos de las variables nominales se expresaron en porcentajes y los obtenidos a partir de variables numéricas se muestran como media y desviación estándar (DE). Al realizar el análisis comparativo de los grupos, la pandemia de influenza A (H1N1) e influenza estacional, para las variables continuas se utilizó la prueba de t-Student, y para las variables discontinuas pruebas de $\chi^{2}$ o de Fisher para valores menores de 5 . El valor $\mathrm{p}<0,05$ se consideró como diferencia estadísticamente significativa.

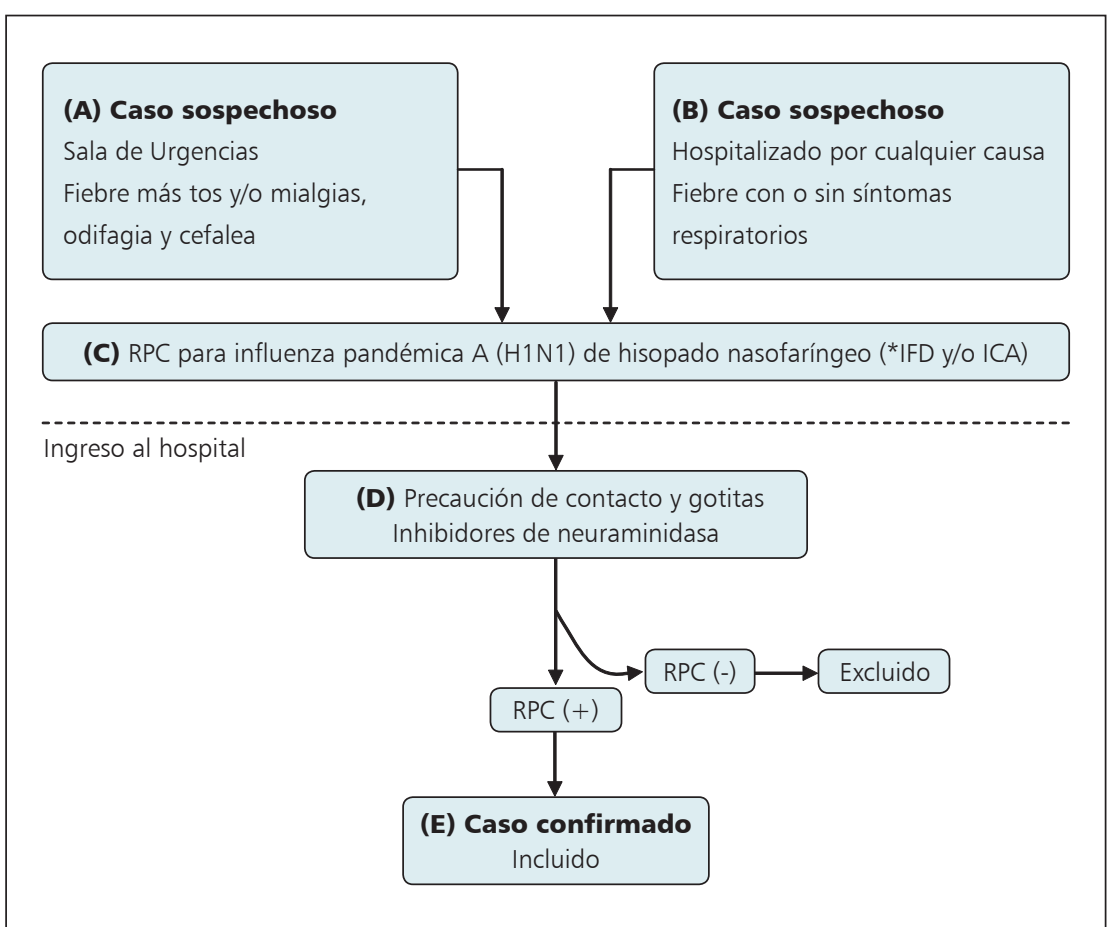

Figura 1. Algoritmo para seleccionar a los pacientes incluidos en la caracterización de la influenza pandémica. Pacientes que consultan en servicio de Urgencia con fiebre y síntomas respiratorios (A) y hospitalizados por cualquier causa que presentaron fiebre con o sin síntomas respiratorios (B), se consideraron sospechosos, fueron sometidos a diagnóstico virológico con reacción de polimerasa en cadena (RPC) de hisopado nasofaríngeo y *otro test si el médico lo requería como análisis inmunocromatográfico (ICA) o immunofluorescencia directa (IFD) (C). Al mismo tiempo, en el momento de ingreso al hospital (línea punteada) para cualquier caso se aplicaron precauciones de contacto y gotitas, y se les prescribió tratamiento antiviral con oseltamivir o zanamivir (D). Los casos en que el resultado de la RPC fue positivo se consideraron como casos confirmados y se incluyeron en el estudio (E). 


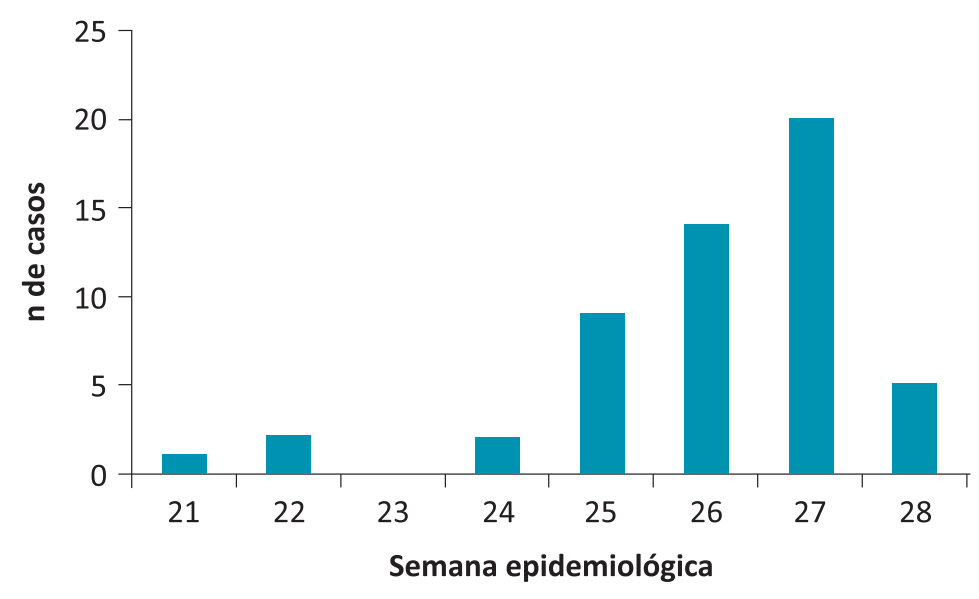

Figura 2. Distribución semanal del número de casos diagnosticados con influenza pandémica A (H1N1) entre el 18 de mayo y 19 de julio 2009 (semanas epidemiológicas 21 a 29).

\begin{tabular}{|c|c|c|}
\hline & $\mathbf{n}$ & $\%$ \\
\hline \multicolumn{3}{|l|}{ Sintomas } \\
\hline Fiebre & 45 & 83,3 \\
\hline Tos & 42 & 77,7 \\
\hline Disnea & 27 & 50,0 \\
\hline Debilidad & 21 & 38,9 \\
\hline Mialgias & 21 & 38,9 \\
\hline Expectoración & 19 & 35,2 \\
\hline Congestión nasal & 14 & 25,9 \\
\hline Odinofagia & 13 & 24,1 \\
\hline Cefalea & 12 & 22,2 \\
\hline Calofríos & 11 & 20,4 \\
\hline Disfonía & 2 & 3,7 \\
\hline Vómitos & 2 & 3,7 \\
\hline Diarrea & 2 & 3,7 \\
\hline \multicolumn{3}{|l|}{ Signos } \\
\hline Crepitaciones & 28 & 51,9 \\
\hline Taquipnea* & 28 & 51,9 \\
\hline Taquicardia** & 23 & 42,6 \\
\hline Roncus y/o sibilancias & 18 & 33 \\
\hline Congestión faríngea & 15 & 27,7 \\
\hline \multicolumn{3}{|c|}{$\begin{array}{l}\text { *Frecuencia respiratoria }>20 \text { respiraciones por min. }{ }^{* *} \text { Frecuenc } \\
\text { cardíaca }>100 \text { latidos por min. }\end{array}$} \\
\hline
\end{tabular}

\section{Resultados}

Se identificó un total de 58 pacientes con influenza pandémica A (H1N1) confirmada; sin embargo, cuatro fueron excluidos por información incompleta. La Figura 2 muestra la distribución temporal de los casos.

Veintiocho $(51,9 \%)$ casos eran género masculino, la edad media era 52,8 $\pm 19,5$ (15 a 90 años de edad). Cuarenta y tres $(79,6 \%)$ tenían una enfermedad crónica: hipertensión arterial $(38,8 \%)$, enfermedad pulmonar obstructiva crónica o asma $(27,7 \%)$, diabetes mellitus $(14,8 \%)$ y cáncer $(14,8 \%)$. Nueve pacientes $(16,7 \%)$ eran inmunocomprometidos: seis estaban bajo tratamiento corticoesteroidal o inmunosupresor, dos con citotóxicos, y uno tenía una infección por VIH. Cuatro $(15,4 \%)$ de los 26 pacientes de género femenino cursaban un embarazo (de 5 a 38 semanas de gestación).

Clínicamente, los síntomas más frecuentes fueron fiebre, tos y disnea; taquipnea, taquicardia y crepitaciones pulmonares los signos más frecuentes (Tabla 1). En el momento de la evaluación clínica inicial, sólo 17 pacientes (31,5\%) cumplieron con la definición de caso sospechoso.

Cuarenta y siete $(87 \%)$ de los pacientes tuvieron una prueba $(+)$ de influenza A, diferente a la RPC. La prueba inmunocromatográfica se realizó en 38 casos y la IFD se realizó en 28 pacientes. La concordancia con la RPC fue de 55,3 y $60,7 \%$ respectivamente.

Los diagnósticos sindromáticos al momento de la hospitalización en los 40 pacientes con influenza pandémica A (H1N1) adquirida en la comunidad fueron: 13 (32,5\%) influenza, $12(30 \%)$ insuficiencia respiratoria y $7(17,5 \%)$ síndrome febril.

En 14 pacientes $(25,9 \%)$ que habían ingresado por otra causa, se diagnosticó influenza pandémica A (H1N1) después de su ingreso por otra causa. Las manifestaciones clínicas comenzaron $16 \pm 16,7$ días (rango 4-66) después de su admisión. Dos de las cuatro pacientes embarazadas desarrollaron influenza durante su hospitalización. Algunas de las diferencias clínicas observadas cuando se compararon los 40 pacientes con adquisición en la comunidad frente a los 14 pacientes con adquisición nosocomial de influenza pandémica $\mathrm{H} 1 \mathrm{~N} 1$ se resumen en la Tabla 2.

Se prescribió oseltamivir en $52(96,3 \%)$ pacientes y zanamivir en $2(3,7 \%)$ casos. Ambos medicamentos fueron bien tolerados y sólo en un caso fue reportado como efecto adverso una leve erupción eritematosa generalizada maculo-papular, probablemente asociada al oseltamivir. Tratamiento empírico con antimcrobianos, cefalosporinas de $3^{\text {a }}$ generación o fluoroquinolonas, fue administrado simultáneamente a 77,7\% de los pacientes, debido a sospecha de sobreinfección bacteriana (bronquitis o neumonía) o debido a la dificultad para descartar la infección bacteriana en pacientes graves. 
La complicación más frecuente fue insuficiencia respiratoria que se observó en 26 pacientes $(48,1 \%)$ : en 10 casos $(18,5 \%)$ fue leve ya que requirieron una fracción inspirada de oxígeno $\left(\mathrm{FiO}_{2}\right)$ menor al 30\%, moderada en 11 casos $(20,3 \%)$ que requirieron $\mathrm{FiO}_{2}$ inferior $50 \%$, y grave en 5 casos $(9,3 \%)$ que requieron $\mathrm{FiO}_{2} \geq 50 \%$ y/o ventilación mecánica ( 2 casos no invasora y 3 casos de ventilación invasora). Comparando los pacientes que requirieron ventilación mecánica vs los que no la requirieron, no se observaron diferencias estadísticamente significativas con respecto a la edad, la frecuencia de enfermedades crónicas o linfopenia; en cambio, presentaban neumonía con mayor frecuencia (100 vs 30,6\%, $\mathrm{p}=0,0004)$ y mayor mortalidad (40 vs $2 \%, p=0,02)$. Ninguna paciente embarazada requirió ventilación mecánica.

Se diagnosticó neumonía en 20 pacientes (37\%), con base en hallazgos anormales en la radiografía de tórax: 12 casos con consolidación pulmonar, en siete de ellos con dos o más focos, tres con infiltrados intersticiales, tres con edema peri-hiliar, uno con distress respiratorio y uno con atelectasias. Se realizaron hemocultivos en 29 pacientes con resultados negativos; también resultaron negativos los cultivos de expectoración llevados a cabo en otros nueve pacientes. Se diagnosticó bacteriemia por Klebsiella pneumoniae en un caso después de siete días del diagnóstico de influenza A (H1N1). Al comparar los casos con versus sin neumonía, se observaron algunas diferencias: los pacientes con neumonía eran mayores $(63,3 \pm 17,3$ vs 46,8 $\pm 18,3$ años, $p=0,002)$, presentaban mayor frecuencia de saturación arterial de oxígeno por debajo del $90 \%$ (50 vs 26,5\%, p = 0,05); hospitalización más prolongada $(15 \pm 15,5$ vs $11,1 \pm 19,9$ días, $\mathrm{p}=0,42)$, y mayor mortalidad (15 vs $0 \%$; $\mathrm{p}=0,045)$

La duración de hospitalización fue de 12,5 \pm 18,4 días (mediana de 5 días, rango de 2-109 días). En promedio, el número de días para tornarse afebril fue 1,74 $\pm 1,63$ días (1 a 8 días).

Otras complicaciones observadas en $19(35,2 \%)$ de los pacientes fueron: insuficiencia renal en seis pacientes $(11,1 \%)$, inestabilidad hemodinámica en cinco $(9,3 \%)$, insuficiencia cardíaca en cuatro $(7,4 \%)$ y delirium en dos $(3,7 \%)$. Sólo una de las cuatro mujeres embarazadas tuvo que ser traslada a la unidad de cuidados intensivos debido a convulsiones secundarias a trombosis del seno venoso, sin poderse establecer una asociación clara con la influenza.

Tres pacientes fallecieron. Uno de estos casos fue una mujer de 37 años de edad con posible vasculitis renal y pulmonar que presentó insuficiencia respiratoria progresiva e irreversible post-influenza, falleciendo dos semanas después del diagnóstico de influenza pandémica A(H1N1). Otro caso fue un hombre de 63 años que estaba bajo quimioterapia por leucemia aguda, que en el contexto
Tabla 2. Comparación de 40 casos adquiridos en la comunidad vs $\mathbf{1 4}$ casos adquiridos en el hospital de influenza pandémica A (H1N1)

\begin{tabular}{|c|c|c|c|}
\hline & $\begin{array}{c}\text { Adquisición en la } \\
\text { comunidad } \\
n=40 \\
\text { Media } \pm \text { DS/ } n(\%)\end{array}$ & $\begin{array}{c}\text { Adquisición } \\
\text { nosocomial } \\
n=14 \\
\text { Media } \pm \text { DS/ } n(\%)\end{array}$ & $\mathbf{P}$ \\
\hline Edad (años) & $51,7 \pm 19,8$ & $53,3 \pm 19$ & 0,78 \\
\hline Sexo masculino & $23(57,5)$ & $5(35,7)$ & 0,09 \\
\hline Fiebre & $31(77,5)$ & $14(100)$ & $<0,001$ \\
\hline Tos & $31(77,5)$ & $11 \quad(78,5)$ & 0,29 \\
\hline Disnea & $23(57,5)$ & $4(28,6)$ & 0,045 \\
\hline Debilidad & $18(45)$ & $3(21,4)$ & 0,07 \\
\hline Mialgias & $20(50)$ & $1 \quad(7,1)$ & 0,003 \\
\hline Crepitaciones & $20(50)$ & $8(57,1)$ & 0,22 \\
\hline Roncus y/o sibilancias & $14(35)$ & $4(28,5)$ & 0,23 \\
\hline Leucocitos (céls/mm³) & $9.292 \pm 5.280$ & $6.628 \pm 3.798$ & 0,05 \\
\hline Linfopenia* & $18(45)$ & $8(57,1)$ & 0,18 \\
\hline $\begin{array}{l}\text { VHS }(\mathrm{mm} / \mathrm{h})^{\star *} \\
\text { (valor normal }<20 \mathrm{~mm} / \mathrm{h} \text { ) }\end{array}$ & $29,4 \pm 25,8$ & $47,5 \pm 31,4$ & 0,06 \\
\hline $\begin{array}{l}\mathrm{PCR}(\mathrm{mg} / \mathrm{dL})^{* * *} \\
(\text { valor normal }<1 \mathrm{mg} / \mathrm{dL} \text { ) }\end{array}$ & $5,2 \pm 4,6$ & $10,3 \pm 7,4$ & 0,03 \\
\hline Neumonía & $12(30)$ & $8(57,1)$ & 0,07 \\
\hline Ingreso a UCl/intermedio & $16(40)$ & $10(71,4)$ & 0,06 \\
\hline Mortalidad & $0 \quad(0)$ & $2(14,3)$ & 0,06 \\
\hline
\end{tabular}

*Linfopenia $<1.000 \mathrm{cel} / \mathrm{mm}^{3}$. **VHS cuantificada en 34 casos con adquisición en la comunidad y 13 casos con adquisición nosocomial. ***PCR cuantificada en 38 casos con adquisición en la comunidad y 14 casos con adquisición nosocomial.

de una neutropenia prolongada presentó sepsis grave con distress respiratorio como evento final en que sólo se demostró influenza pandémica A (H1N1). Un tercer caso fue un hombre de 75 años de edad sin enfermedades crónicas, que se recuperó de la infección viral, pero más tarde sufrió una hemorragia digestiva grave. Sobre estos hechos la influenza pandémica A (H1N1) fue parte de la cadena de eventos que condujeron a la muerte en sólo dos casos, por lo que la mortalidad fue de $3,7 \%$.

En cuanto a la comparación entre la influenza pandémica A (H1N1) y estacional (Tabla 3), se observó que la primera afectó a un menor número de pacientes mayores. De hecho, la frecuencia de pacientes $\geq 65$ años de edad diagnosticados con influenza pandémica A (H1N1) fue de $31,5 \%$ frente al $68 \%$ diagnosticados con influenza estacional $(\mathrm{p}<0,0001)$. Una gran proporción de pacientes con enfermedades crónicas y un número relativamente bajo de los sujetos inmunocomprometidos se observó en ambos grupos. Sólo se observaron mujeres embarazadas hospitalizadas a causa de la influenza pandémica A (H1N1) durante el brote actual. Se duplicó el número 
Tabla 3. Comparación de las características clínicas y epidemiológicas entre 54 pacientes con influenza pandémica A (H1N1) vs 95 pacientes con influenza estacional (1999 y 2004)

\begin{tabular}{|c|c|c|c|}
\hline & $\begin{array}{c}\text { Influenza pandémica } \\
\qquad \begin{array}{l}n=54 \\
\text { Media } \pm D S / n(\%)\end{array}\end{array}$ & $\begin{array}{c}\text { Influenza estacional } \\
\qquad \begin{array}{c}n=95 \\
\text { Media } \pm D S / n(\%)\end{array}\end{array}$ & $\mathbf{p}$ \\
\hline Edad (años) & $52,9 \pm 19,5$ & $63,2 \pm 21,5$ & 0,003 \\
\hline Sexo masculino & $28(51,9)$ & $44(46,3)$ & 0,5 \\
\hline Enfermedades crónicas & $43(79,6)$ & $67(70,5)$ & 0,2 \\
\hline Adquisición nosocomial & $14(25,9)$ & $12(12,6)$ & 0,04 \\
\hline Fiebre $>38^{\circ} \mathrm{C}$ axilar & $32(59,3)$ & $74(77,9)$ & 0,02 \\
\hline Calofríos & $11(20,4)$ & $58(61,1)$ & $<0,001$ \\
\hline Mialgia & $21(38,9)$ & $59(62,1)$ & 0,006 \\
\hline Tos & $42(77,7)$ & $89(93,7)$ & 0,004 \\
\hline Expectoración & $19(35,2)$ & $65(68,4)$ & $<0,001$ \\
\hline Disnea & $27(50)$ & $46(48,4)$ & 0,8 \\
\hline Congestión nasal & $14(25,9)$ & $32(33,7)$ & 0,3 \\
\hline Odinofagia & $13(24,1)$ & $19(20)$ & 0,5 \\
\hline Cefalea & $12(22,2)$ & $37(38,9)$ & 0,04 \\
\hline Vómitos & $2(3,7)$ & $18(18,9)$ & 0,01 \\
\hline Crepitaciones & $28(51,9)$ & $54(56,8)$ & 0,5 \\
\hline Obstrucción bronquial & $18(33,3)$ & $48(50,5)$ & 0,04 \\
\hline Leucocitos (céls/mm³) & $8.601 \pm 5.044$ & $9.734 \pm 4.247$ & 0,16 \\
\hline Baciliformes $>5 \%$ & $3(5,6)$ & $54(56,8)$ & $<0,001$ \\
\hline $\begin{array}{l}\mathrm{PCR}(\mathrm{mg} / \mathrm{dL}) \text { (Valor normal } \\
<1 \mathrm{mg} / \mathrm{dL})\end{array}$ & $6,6 \pm 5,9$ & $9,4 \pm 6,5$ & 0,008 \\
\hline $\begin{array}{l}\text { VHS }(\mathrm{mm} / \mathrm{h}) \text { (Valor normal } \\
<20 \mathrm{~mm} / \mathrm{h})\end{array}$ & $34,4 \pm 28,3$ & $37,8 \pm 28,6$ & 0,48 \\
\hline Neumonía & $20(37)$ & $22(23,2)$ & 0,07 \\
\hline Ingreso a UCl/ intermedio & $26(48,1)$ & $24(25,3)$ & 0,006 \\
\hline Mortalidad & $2(3,7)$ & $1(1,1)$ & 0,6 \\
\hline
\end{tabular}

de casos de influenza A(H1N1) adquiridos en el hospital durante la pandemia comparado con los anteriores brotes de influenza estacional. Los síntomas clínicos presentados por los pacientes con influenza pandémica $\mathrm{A}(\mathrm{H} 1 \mathrm{~N} 1)$ con frecuencia no se ajustaba al síndrome gripal clásico, presentaban menos fiebre y signos de obstrucción bronquial. Por último, se observó más casos de neumonía, admisión en unidades de cuidados intensivos e intermedios y mortalidad entre los pacientes con influenza pandémica A (H1N1) en comparación con los casos de influenza estacional.

\section{Discusión}

En este estudio se incluyeron 54 adultos hospitalizados con infección confirmada por virus influenza pandémico
A (H1N1). Los pacientes mostraron una amplia gama de signos y síntomas, que van desde la fiebre aislada, hasta cuadros de neumonía e insuficiencia respiratoria grave. Un tercio de los pacientes requirió traslado a unidades de cuidados intermedios e intensivos. Alrededor de $25 \%$ de los pacientes fueron casos con adquisición nosocomial. La letalidad de $3,7 \%$, fue casi cuatro veces mayor a la observada entre los pacientes hospitalizados en nuestro centro hace 5 y 10 años durante la actividad de influenza estacional. Es importante destacar que la actividad del virus de la influenza pandémica A (H1N1) observada en nuestro centro es similar a la actividad de influenza en todo Chile que se informó a las autoridades nacionales de salud ${ }^{14}$.

En diferentes series publicadas, se indicó que la influenza pandémica A (H1N1) afectó a un grupo de menor edad que lo habitual en influenza ${ }^{4,6}$. De hecho, en una comparación entre influenza estacional y pandémica recientemente publicada por Shiley y cols., destaca que la mediana de edad en la primera fue 59 vs 29 años en la segunda, correspondiendo a una diferencia estadísticamente significativa ${ }^{15}$. Esto también fue confirmado en nuestras observaciones, ya que la edad media de los pacientes con influenza pandémica A (H1N1) fue significativamente menor que los pacientes de la influenza estacional. Hancock y cols., exploraron las razones por las que fue afectado un grupo de menor edad, identificando anticuerpos de reactividad cruzada contra la influenza pandémica $\mathrm{A}(\mathrm{H} 1 \mathrm{~N} 1)$ en $4 \%$ de la población nacida después de 1980 frente a $34 \%$ en los nacidos antes de 1950, lo que sugiere la exposición a otro virus de influenza A (H1N1) similar al que causó la pandemia de $1918^{16}$. Por otro lado, al igual que nuestros datos, en el grupo que incluyó pacientes de edad avanzada y pacientes con co-morbilidades se observó el desarrollo de neumonía como complicación más frecuentemente ${ }^{6}$. La alta frecuencia de co-morbilidades es común entre los pacientes con influenza estacional que requieren hospitalización ${ }^{7,17}$. Esto ya ha sido descrito en otras series de influenza pandémica A (H1N1), donde las co-morbilidades más frecuentes son: enfermedad pulmonar obstructiva crónica, asma, insuficiencia cardíaca congestiva, inmunodeficiencias, diabetes mellitus y obesidad $^{4,18}$. Lamentablemente, el índice de masa corporal no está disponible en los datos del presente estudio ni en los de la serie anterior de influenza estacional, que serían necesarias para corroborar las observaciones de que los casos más graves se observaron en los pacientes obesos ${ }^{19}$.

Se observaron más pacientes embarazadas con influenza A (H1N1) en comparación con nuestra serie anterior de influenza estacional, hecho también señalado por Shiley y cols ${ }^{15}$. Esto podría explicarse en parte por el hecho de que se vio afectado principalmente un grupo de menor edad. Además, apoya el hecho de que las mujeres 
embarazadas son particularmente más susceptibles al desarrollo de influenza y sus complicaciones ${ }^{20}$. Treinta y cuatro pacientes fueron descritas a partir de una serie de mujeres embarazadas con influenza A (H1N1) en E.U.A. La mayoría estaba en su primer o segundo trimestre de embarazo, 26,5\% presentaban co-morbilidades y $11 \%$ requirió hospitalización, que era mayor a lo observado en la población general $(0,32 / 100.000$ mujeres embarazadas vs 0,076/100.000 población general en riesgo $)^{21}$. Esta información hace que sea fundamental considerar sobre todo este grupo en términos de estrategias de prevención que deben aplicarse en la siguiente temporada de influenza.

El número de pacientes cuyos síntomas clínicos correspondían a la definición de caso sospechoso por cuadro clínico de influenza fue relativamente bajo, alcanzando sólo a $31,5 \%$. Esto pudo ser debido a la amplia gama de signos y síntomas, desde fiebre leve a insuficiencia respiratoria catastrófica. Este hecho destaca que la estrategia de diagnóstico es particularmente relevante para los casos nosocomiales de influenza A (H1N1), ya que sólo una quinta parte de estos pacientes cumplió los criterios de síndrome gripal. En base a esto, se recomienda la aplicación de una estrategia de sospecha amplia, especialmente entre los pacientes que deben ser sometidos a test confirmatorios para influenza pandémica $\mathrm{A}(\mathrm{H} 1 \mathrm{~N} 1)$, sobre todo cuando presenten fiebre sin otra causa o cuando la fiebre se acompañe de un mínimo de síntomas respiratorios durante la temporada de influenza.

En cuanto al diagnóstico etiológico, la sensibilidad de las pruebas de inmunocromatografía e IFD fueron inferiores a $60 \%$ en comparación con la RPC; resultados similares han sido publicados por otros autores ${ }^{6,22}$. En otras palabras, hasta $40 \%$ de los casos va a tener un falso negativo como resultado con estas pruebas. Por lo tanto, se recomienda el uso de la técnica de RPC además de las otras pruebas de diagnóstico rápido.

Un aspecto destacado recientemente por nuestro gru$\mathrm{po}^{23}$, fue la mayor tasa de influenza $\mathrm{A}(\mathrm{H} 1 \mathrm{~N} 1)$ adquirida de forma nosocomial, que podría reflejar mayor circulación del virus no sólo entre los pacientes, sino también entre los visitantes y trabajadores de la salud; también podría ser un reflejo de una mayor transmisibilidad del virus o, por el contrario, podría ser consecuencia de un umbral más bajo aplicado al considerar el diagnóstico y la amplia disponibilidad de técnicas de diagnóstico de laboratorio sensibles. Con el fin de prevenir la transmisión nosocomial de la pandemia de influenza A (H1N1), se aplicó una serie de acciones nuevas en nuestro hospital como una campaña de comunicación orientada a familiares y amigos que visitaban el centro hospitalario, la restricción de visitantes sintomáticos respiratorios; el manejo de todos los casos con precauciones de contacto y gotitas, además del tratamiento antiviral temprano en los casos sospechosos después de obtener las muestras correspondientes. Además, las hospitalizaciones por cirugías electivas fueron canceladas durante las dos semanas de mayor actividad de la pandemia. Es imposible determinar si estas acciones fueron eficaces y la forma en que ayudó a reducir la transmisibilidad. Cabe señalar que los pacientes con influenza $\mathrm{A}(\mathrm{H} 1 \mathrm{~N} 1)$ nosocomial mostraron tasas más altas de neumonía, admisión a unidades de cuidados intensivos y mortalidad. Todo esto confirma la complejidad de la influenza nosocomial ${ }^{24}$ y demuestra la importancia de establecer una política de control estricto de la infección durante toda la temporada del brote de influenza.

El índice de desarrollo de neumonía fue mayor que el observado para la influenza estacional aunque no fue estadísticamente significativo. En otras series de pacientes hospitalizados con influenza pandémica $\mathrm{A}(\mathrm{H} 1 \mathrm{~N} 1)$, la tasa de frecuencia de neumonía radiológica uni o multilobar fue de $60 \%{ }^{4,21}$. Dichos casos de neumonía podrían haber sido de etiología exclusivamente viral, en base a los resultados bacteriológicos negativos. Por otra parte, teniendo en cuenta los datos actuales publicados sobre neumonía viral con patrones uni o multilobar, se puede sugerir que varios de los casos descritos en esta serie podrían haber sido secundarios a influenza. Sin embargo, en la práctica clínica, es imposible descartar una neumonía bacteriana y evitar el uso de antimicrobianos en pacientes con enfermedad grave.

Un punto a considerar, es que las diferencias observadas en nuestra comparación sobre la influenza pandémica A (H1N1) y la influenza estacional podrían explicarse por los diferentes patrones de infección entre los virus A (H1N1) y A (H3N2). Kelly y cols., compararon los casos de influenza A (H1N1) de origen humano con los de la influenza pandémica $\mathrm{A}(\mathrm{H} 1 \mathrm{~N} 1)$ de origen porcino, demostrando que ambas infecciones afectaron principalmente a un grupo más joven ${ }^{25}$. Es necesario tener más información para comprender mejor las diferencias entre el nuevo virus $\mathrm{A}(\mathrm{H} 1 \mathrm{~N} 1)$ y los virus estacionales $\mathrm{A}(\mathrm{H} 1 \mathrm{~N} 1)$ o $\mathrm{A}$ (H3N2). No pudimos responder a esta pregunta, porque no estábamos seguros si todos los casos de influenza A estacional que se incluyeron sólo correspondían al virus A (H3N2) o algunos de ellos eran A (H1N1).

La prescripción precoz de un antiviral fue un punto esencial en la gestión de la pandemia de infecciones de influenza A (H1N1). El resultado general observado en esta serie debe ser evaluado teniendo en cuenta el uso de medicamentos antivirales en todos los casos. Según un reciente estudio realizado por Jain y cols., es muy recomendable el inicio de los medicamentos antivirales en las primeras 48 horas después de iniciarse los síntomas. Esto se basa en el hecho de que los pacientes que fueron ingresados en una UCI o murieron tenían menos probabilidad de haber recibido tratamiento antiviral a tiempo ${ }^{4}$. Del mismo modo, en datos recientemente publicados 
de otro centro de Santiago, un grupo de 129 pacientes hospitalizados con un promedio de edad de 19 años, que recibieron inhibidores de la neuraminidasa en las primeras 48 horas de iniciados los síntomas, no presentaron muertes asociadas a la influenza, explicado posiblemente por el uso precoz de medicamentos antivirales ${ }^{26}$.

La principal fortaleza de este estudio es que sólo se consideran los casos probados de influenza pandémica A (H1N1) y se incluyó una comparación con los casos de influenza estacional manejados en el mismo hospital por el mismo grupo de investigación. Entre sus limitaciones destaca: algunos casos no se incluyeron en este estudio debido a la falta de la confirmación por análisis de RPC y los pacientes del grupo de comparación de influenza estacional fueron diagnosticados con métodos no moleculares.

En resumen, la pandemia de infección por el virus influenza A (H1N1) en adultos hospitalizados se caracterizó por comprometer un grupo de edad más joven, a pacientes con co-morbilidad, presentar una elevada transmisión nosocomial a pesar de las medidas de control y una alta frecuencia de neumonía y mortalidad en comparación con personas infectadas con influenza estacional durante temporadas anteriores.

\section{Resumen}

La pandemia de influenza A (H1N1) 2009 generó preguntas sobre sus diferencias con influenza estacional. $\mathrm{Ob}$ jetivos: Describir las características de influenza pandémica y comparar con influenza estacional. Pacientes y Métodos: Estudio descriptivo de casos confirmados de influenza pandémica en adultos internados en el Hospital Clínico de la Pontificia Universidad Católica entre mayo y julio de 2009, comparado con 95 casos históricos de influenza estacional. Resultados: 54 pacientes con influenza pandémica, 51,9\% género masculino, edad 52,8 $\pm 19,5$ años; $79,6 \%$ presentaban co-morbilidades; $16,7 \%$ inmunocomprometidos, 7,4\% mujeres embarazadas, $25,9 \%$ de adquisición nosocomial, $31,5 \%$ requirió cuidados intensivos/intermedios. Se diagnosticó neumonía en 37\% y la mortalidad global fue 3,7\%. En la comparación con influenza estacional, la pandémica afectó menos pacientes $\geq$ de 65 años $(31,5$ vs $68 \%$, p < 0,0001), dobló los casos con adquisición nosocomial y hubo más casos de neumonía y muertes. Conclusiones: La infección por influenza pandémica afectó a un grupo de menor edad y generó mayor transmisión nosocomial, neumonía y muerte que la influenza estacional.

\section{Referencias}

1.- Novel swine-origin influenza A (H1N1) Virus Investigation Team, Dawood FS, Jain S, et al. Emergence of a novel swine-origin influenza A (H1N1) virus in humans. N Engl J Med 2009; 360: 2605-15.

2.- Pandemic influenza (H1N1) 2009. Ministry of Health of Chile Report 31 May 2009. http:// www.redsalud.gov.cl/portal/url/item/6b64ba57c bfea979e04001011f01072f.pdf (Accedido 17 de marzo de 2010).

3.- Centers for Disease Control and Prevention (CDC). Hospitalized patients with novel influenza A (H1N1) virus infection - California, April-May, 2009. MMWR Morb Mortal Wkly Rep 2009; 58: 536-41.

4.- Jain S, Kamimoto L, Bramley A M, Schmitz A M, Benoit S R, Louie J, et al. Hospitalized patients with 2009 H1N1 influenza in the United States, April-June 2009. N Engl J Med October 2009 361: 1935-4.

5.- Pérez-Padilla R, de la Rosa-Zamboni D, Ponce de León S, Hernández M, QuiñonesFalconi F, Bautista E, et al. Pneumonia and respiratory failure from swine-origin influenza A (H1N1) in Mexico. N Engl J Med 2009; 361: 680-9.

6.- Human infection with pandemic A (H1N1) 2009 influenza virus: clinical observations in hospitalized patients, Americas, July 2009-update. Wkly Epidemiol Rec 2009; 84: 305-8.

7.- Baker M G, Kelly H, Wilson N. Pandemic H1N1 influenza lessons from the southern hemisphere. Euro Surveill. 2009; 14 (42): pii $=19370$. disponible en URL: http:// www.eurosurveillance.org/ViewArticle. aspx?ArticleId=19370.

8.- Murata Y, Walsh E E, Falsey A R. Pulmonary complications of interpandemic influenza A in hospitalized adults. J Infect Dis 2007; 195: 1029-37.

9.- Clinical guidelines to case management new human influenza A H1N1 (IHA H1N1). Pandemic phase. Version 2.3. Division of Disease Prevention and Control. Ministry of Health of Chile. Acceso Octubre 1, 2010; disponible en: http://www.pandemia.cl/pagnew/ profsalud/Guia_clinica_Manejo_de_caso.pdf

10.- Rabagliati R, Benítez R, Fernández A, Gaete P, Guzmán AM, Garcia P et al. Influenza-A as etiology of fever and respiratory insufficiency in adults hospitalized during an outbreak in Chile. Rev Méd Chile 2004; 132 : 317-24.

11.- Rabagliati B R, Serri V M, Perret P C, Guzmán A M, Azócar A T, Habash A L, et al. Clinical and epidemiological characteristics of respiratory virus infections among adults hospitalized during 2004 influenza season. Rev Chil Infectol 2006; 23: 111-7.

12.- Fasce R. Vigilancia de Laboratorio Influenza.
Chile 1998-1999. El vigia. Boletin de Vigilancia Epidemiológica de Chile. Ministery of Health of Chile. 1999; 10: 7-8. (Acceso febrero 16, 2011) disponible en: http://epi.minsal.cl/epi/html/ elvigia/Vigia10.pdf

13.- Sotomayor V. Influenza. El vigia. Boletin de Vigilancia Epidemiológica de Chile. Ministery of Health of Chile. 2004; 22: 42-43. (Accedido 16 de febrero de 2011) disponible en: http://epi.minsal.cl/epi/html/elvigia/Vigia22. pdf

14.- Pandemic influenza (H1N1) 2009. Ministry of Health of Chile Report October 7, 2009. Acceso Octubre 13, 2010; disponible en: http://www. redsalud.gov.cl/minsalaudios/reporte6octubre. pdf

15.- Shiley K T, Nadolski G, Mickus T, Fishman N O, Lautenbach E. Differences in the epidemiological characteristics and clinical outcomes of pandemic (H1N1) 2009 influenza, compared with seasonal influenza. Infect Control Hosp Epidemiol 2010; 31: 676-82.

16.- Hancock K, Veguilla V, Lu X, Zhong W, Butler E N, Sun H, et al. Cross-reactive antibody responses to the 2009 pandemic H1N1 influenza virus. N Engl J Med 2009; 361: 1945-52.

17.- Harper S A, Bradley J S, Englund J A, File T M, Gravenstein S, Hayden F G, et al. Seasonal influenza in adults and children-diagnosis, treatment, chemoprophylaxis, and institutional outbreak management: Clinical practice 
Guidelines of the Infectious Diseases Society of America. Clin Infect Dis 2009; 48: 1003-32.

18.- Rello J, Rodríguez A, Ibáñez P, Socias L, Cebrian J, Marques A, et al. Intensive care adult patients with severe respiratory failure caused by influenza A (H1N1)v in Spain. Crit Care. 2009; 13: R148. Epub 2009 Sep 11.

19.- Centers for Disease Control and Prevention (CDC). Intensive-care patients with severe novel influenza A (H1N1) virus infectionMichigan, June 2009. MMWR Morb Mortal Wkly Rep 2009; 58: 749-52.

20.- Neuzil K M, Reed G W, Mitchel E F, Simonsen L, Griffin M R. Impact of influenza on acute cardiopulmonary hospitalizations in pregnant women. Am J Epidemiol 1998; 148 : 1094-102.

21.- Jamieson D J, Honein M A, Rasmussen S A, Williams J L, Swerdlow D L, Biggerstaff M S, et al. H1N1 2009 influenza virus infection during pregnancy in the USA. Lancet 2009; 374: 451-8.

22.- Vasoo S, Stevens J, Singh K. Rapid antigen tests for diagnosis of pandemic (swine) influenza A/ H1N1. Clin Infect Dis 2009; 49 (7): 1090-3.

23.- Rabagliati R, Labarca J, Siri L, Pérez C M, Ferrés M. Rates of hospital-acquired influenza due to the pandemic H1N1 virus in 2009, compared with seasonal influenza. Infect Control Hosp Epidemiol 2011; 32: 198-200
24.- Salgado C D, Farr B M, Hall K K, Hayden F G. Influenza in the acute hospital setting. Lancet Infect Dis 2002; 2: 145-55.

25.- Kelly H, Grant K, Williams S, Smith D. H1N1 swine origin influenza infection in the United States and Europe in 2009 may be similar to H1N1 seasonal influenza infection in two Australian states in 2007 and 2008. Influenza Other Respi Viruses 2009; 3: 183-8.

26.- Torres J P, O’Ryan M, Herve B, Espinoza R, Acuña G, Mañalich J, et al. Impact of the novel influenza A (H1N1) during the 2009 autumn-winter season in a large hospital setting in Santiago, Chile. Clin Infect Dis 2010; 50: 860-8. 